\title{
Sepsis in HIV-positive patients. What is the scale of the problem and how to approach it?
}

\author{
Dagny C. Krankowska, Tomasz Mikuła, Alicja Wiercińska-Drapało \\ Department of Infectious and Tropical Diseases and Hepatology, Medical University of Warsaw, Poland
}

\begin{abstract}
In the antiretroviral therapy (ART) era, human immunodeficiency virus (HIV)-positive patients are admitted to the hospital in a critical state for various reasons, with a decrease of hospitalizations due to opportunistic infections. About 12-31\% of hospitalizations of HIV-infected patients in the intensive care unit (ICU) are due to sepsis. HIV-positive patients with sepsis have a higher mortality rate than HIV-negative patients. Although there are no specific recommendations on how to treat septic HIV-positive patients, it is important to remember which pathogens are the most common causes of infection in this group of patients. More attention should be drawn to nosocomial infections mainly caused by Gram-positive cocci and Gram-negative rods. According to the European AIDS Clinical Society Guidelines (EACS) 2015, ART should be introduced in all HIV-positive patients, regardless of the CD4 cell count. The recommendations do not contain information on treatment of patients with HIV with sepsis. The introduction of ART in HIV-positive patients in a critical state is still debatable due to the drug-drug interactions and route of administration. Though ART in critically ill patients may not affect the hospital survival, it has long-term benefits such as fewer AIDS-related events. The aim of this article was to provide physicians with an overview of recent information on patients with HIV who develop sepsis.
\end{abstract}

HIV AIDS Rev 2017; 16: 1-4 DOI: https://doi.org/10.5114/hivar.2017.65333

Key words: sepsis, HIV, ART, nosocomial infections.

\section{Introduction}

According to the Third International Consensus Definition for Sepsis and Septic Shock (Sepsis-3), set in February 2016, sepsis should be defined as 'life-threatening organ dysfunction caused by a dysregulated host response to infection' [1]. Sepsis incidence in the United States increased from 621000 in the year 2000 to 1141000 in 2008 [2]. According to some other data, it is estimated that sepsis incidence is about 150-300 cases per 100000 inhabitants. The incidence increases up to 700/100 000 in patients with chronic diseases and up to $1000 / 100000$ in patients with human immunodeficiency virus (HIV) [3]. The incidence of septic shock is estimated to be about 11 cases per 100000 inhabitants. Mortality rates are considered to be up to $50 \%$ for sepsis and $80 \%$ for septic shock. One in 1000 people in developed countries suffer sepsis each year. There are no data about sepsis in developing countries [4]. One percent to $10 \%$ of patients with sepsis have HIV/AIDS and about $12-31 \%$ of admissions of HIV-infected patients to the intensive care unit (ICU) are due to sepsis $[5,6]$.

\section{HIV and sepsis}

There are numerous causes of admission of HIV-positive patients in a critical state to a hospital. They can be either HIV-related (opportunistic) or not (trauma, acute infections). With antiretroviral therapy (ART) becoming more popular, sepsis has become a more frequent diagnosis in HIV-positive

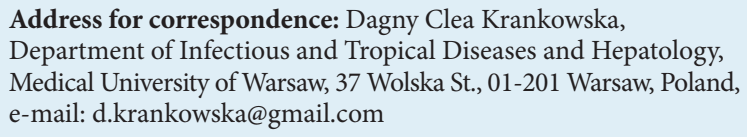

Address for correspondence: Dagny Clea Krankowska, Department of Infectious and Tropical Diseases and Hepatology, Medical University of Warsaw, 37 Wolska St., 01-201 Warsaw, Poland, e-mail:d.krankowska@gmail.com

Article history:

Received: 13.08 .2016

Received in revised form: 30.10 .2016

Accepted: 01.11.2016

Available online: 20.01.2017
International Journal of HIV-Related Problems

HIV \& AIDS

R e vi e w 
patients. A prospective study conducted by Casalino et al. between 1995 and 1999 in Paris showed that after the introduction of ART (in 1997), AIDS-related admissions to the ICU decreased from $57.7 \%$ to $37 \%$, and the opposite trend was seen in septic admissions: the percentage of patients with HIV and sepsis admitted to the ICU increased from $16.3 \%$ to $22.6 \%$ [7]. Even though opportunistic diseases are a decreasing cause of patient hospitalization, respiratory diseases still play a substantial role. In a retrospective study conducted by Turtle et al. on $43 \mathrm{HIV}$ positive patients admitted to the ICU between 2001 and 2006, the majority were admitted due to respiratory disease [8].

The frequencies of bacteria causing pulmonary infections differ between countries and hospitals, but in general the most common pathogens are considered to be Streptococcus pneumoniae, Haemophilus influenzae, Staphylococcus aureus and Pseudomonas aeruginosa. The latter occurs especially in patients with a low CD4 cell count. Fungal (especially caused by Pneumocystis jiroveci) and mycobacterial pneumonias also occur. There has been an increase in hepatic (especially in patients co-infected with HCV), renal and cardiovascular causes of admission of HIV-positive patients [9].

In a study conducted by Greenberg et al. of 990 patients with sepsis, 136 (13.7\%) of them had HIV. Of the latter, $22 \%$ were on ART prior to hospitalization. There were 194 acute infections with 112 nosocomial/health care-associated infections, 55 AIDS-related, and 27 community-acquired infections. The most common pathogen of the communityacquired infections was Streptococcus pneumoniae. Fifty-one percent of the AIDS-related infections were Pneumocystis jirovecii pneumonia (PCP), whereas the nosocomial infections were caused mainly by Gram-positive cocci and Gramnegative rods. Patients with nosocomial/healthcare-associated or AIDS-related infections had lower CD4 counts and were less likely to be on ART $(p<0.05)$. The in-hospital mortality rate was $42 \%$, and it was more associated with acute illness severity than with markers of immunodeficiency [10]. Due to immunosuppression, recurrent antibiotic treatment and more frequent exposure to invasive procedures, HIV-positive patients have a higher risk of nosocomial infections than HIV-negative patients. The incidence of nosocomial infections in HIV-positive patients is estimated to be 7.9-15 per 100 admissions. The main source of infection is through the intravascular catheter [11].

Interestingly, according to Mrus et al., based on a study of discharge abstracts of 74020 patients with sepsis in the year 1999 (10.3\% of them had HIV/AIDS), patients with sepsis and HIV/AIDS were less likely to be admitted to the ICU (37\% vs. $56 \%$ ), but had greater mortality rates than those without HIV/AIDS ( $29 \%$ vs. $20 \%$ ). The comparison of characteristics of the groups of patients with sepsis showed that those with HIV/AIDS and sepsis were younger on average (41.9 years vs. 49.9 years), were less likely to have other comorbidities (39\% vs. $51 \%$ ), but were more likely to be male ( $66 \%$ vs. $54 \%$ ), and more likely to have respiratory and opportunistic infections than those without HIV/AIDS (53\% vs. 9\%) [12].
Similar results were demonstrated in an observational data analysis done by Akgun et al. Here, 539 HIV-positive patients and 375 seronegative patients with organ dysfunction were compared. Patients with HIV were younger and less frequently had hypertension, congestive heart failure, COPD and diabetes. They more frequently had pneumonia and other infectious diseases ( $21 \%$ vs. $12 \%)$, more frequently had mechanical ventilation ( $17 \%$ vs. $9 \%$ ) and had a higher mortality rate ( $18.6 \%$ vs. $11.2 \%)$ than other patients in a critical state without HIV [13].

Another study, done in the Instituto de Pesquisa Clinica Evandro Chagas (IPEC) in Brazil between 2006 and 2008, showed a significantly different hospital mortality of HIVpositive patients with and without sepsis (66\% vs. $34 \%$, $p=0.002)$. The most common sites of infections were: lungs (52\%), primary bloodstream (38\%), venous catheter-related (7\%) and urinary tract (3\%). Ninety percent of the infections were nosocomial. The leading pathogens were: Pseudomonas aeruginosa, Klebsiella pneumoniae, Enterobacter sp., Escherichia coli, Acinetobacter, Serratia marcescens, Staphylococcus sp., and Mycobacterium tuberculosis. By using a Cox proportional hazards regression, a 4 times higher risk of death at 28 days was obtained in septic patients than for non-septic patients [5].

In a consecutive prospective analysis, by Silva et al., even though the severity of sepsis of patients with HIV was similar to that in non-HIV patients and the prognostic scores did not differ much between the two groups, the risk of death at 28 days and at 6 months of follow-up was again higher for HIV-positive patients. The two groups also varied in the etiologies of sepsis - in HIV-positive patients it was mainly fungal and mycobacterial pulmonary or abdominal infections, while in the non-HIV ones it was mainly Gram-positive cocci and Gram-negative rods. Respiratory (77.8\%), cardiovascular (75\%) and neurological (52.8\%) were the main organic dysfunctions in HIV-positive patients, in contrast with cardiovascular $(72.7 \%)$, renal (63.6\%) and lactic acidosis $(29.1 \%)$ in the non-HIV septic patients [11].

In a retrospective study by Medrano et al., HIV-positive patients in the ICU had a higher frequency of sepsis than the control group (non-HIV and non-HCV patients), 57.7\% vs. $39.4 \%$. HIV-infected patients had a higher mortality than the control group, independently of sepsis. The HIV/ HCV-coinfected patients had a higher mortality rate regardless of the presence of sepsis at days 7 and 30, but not 90 [14].

\section{Continue, stop or introduce ART in a HIV-positive patient with severe sepsis?}

Treatment of HIV-positive patients with sepsis does not differ much from treatment of HIV-negative patients with sepsis. In both cases the medical staff should follow the international "Surviving Sepsis Campaign (SSC) Guidelines for Management of Severe Sepsis and Septic Shock" revised in 2012 (the 2016 version will appear soon) [15]. According to the new French 2013 Guidelines for Antiretroviral Ther- 
apy of HIV-1 Infection in Adults as well as of the European AIDS Clinical Society Guidelines 2015, ART should be initiated in any HIV-positive person, regardless of the CD4 cell count $[9,16]$. The limitation to this recommendation is that patients in a critical state are not always able to take the antiretroviral drugs orally and only zidovudine can be administered intravenously. The EACS guidelines do not specify the treatment of an HIV-positive patient with severe sepsis.

Lanoix et al. in the article "Antiretroviral therapy in intensive care" list other issues that need to be taken into consideration when using ART in a patient in a critical state. Namely, the drug interactions, risk of immune reconstitution syndrome (especially when ART is introduced in patients with a CD4+ T-cell count $<100$ cells/ $\mu$ and with a viral load $>100000$ copies/ $\mu$ l) and drug kinetics in patients on mechanical ventilation and/or with gastric tubes [17]. According to Masur, due to the interactions of ART with other drugs metabolized by the cytochrome P-450 enzyme system, and due to the unpredictable pharmacokinetics of ART in severely ill patients (due to poor enteral absorption), in most situations it is best to stop the ART at the admission to the patient and consult an experienced pharmacokineticist and infectious disease specialist [18]. However, stopping the treatment suddenly may greatly increase the immunosuppression of the patient [8].

Casalino et al. compared the survival rates of HIV-infected patients before the ART era and after (between 1995 and 1999). The data showed no difference in ICU mortality between those two eras, whereas the long-term survival rate has increased substantially in the ART era [7]. According to van Lelyveld the 1-year mortality decreased from $71 \%$ in the pre-HAART era to $50 \%$ in the recent period $(p=0.06)$. The 5 -year mortality decreased from $87 \%$ in the pre-HAART era to $59 \%$ in the early-HAART period $(p=0.005)$ [19].

On the other hand, a retrospective study by Dickson $e t$ al. did not show a better outcome in patients receiving ART (although in this study no patient began ART at admission) [20]. Similarly, a prospective study conducted by Japiassu et al. showed that ART use did not affect hospital survival, leaving the mortality rate at $50 \%$ both in those who received ART and those who did not [5]. A Taiwanese univariable analysis of hospital mortality for HIV-positive patients showed no difference between survivors and non-survivors with respect to ART use prior to the hospital admission $(p=0.5)$ or to the introduction of ART at admission $(p=0.9)$ [21]. Similar results were obtained by Meybeck et al. in a retrospective cohort study where $85 \mathrm{HIV}$-infected patients in the ICU were diagnosed. There was no significant difference in the ICU survival or the 6-month survival in patients on ART or not. But the prescription of ART during hospital stay was associated with less frequent AIDS-related events [22]. The reduction of frequency of progression of AIDS was also noted in a study by Zolopa et al., when patients received the ART immediately rather than after opportunistic infection treatment was completed [23]. A more recent study conducted between 2011 and 2013 by Orsini et al. did not question long-term benefits of the use of ART but again did not show any short-term benefits of such therapy [24]. The use of ART did not affect the mortality rate in patients in a critical state. The limitation of both Chiang's and Orsini's studies on the use of ART and the survival rate of patients was that patients' adherence to the therapy prior to hospital admission was not taken into consideration, nor was the prevalence of the mutations responsible for antiretroviral drug resistance.

\section{Conclusions}

Sepsis is still an important global burden. The incidence of sepsis increases in patients with HIV and increases the mortality rate in HIV patients. Since there are no additional recommendations on how to treat HIV-positive patients with sepsis, it is important to remember which pathogens are more often found in those patients. Special attention should be paid to nosocomial infections. Numerous articles analyze the benefits and disadvantages of introducing ART in patients in intensive care units. While there is no conclusive result on whether ART brings immediate, short-term benefits, it is agreed that it brings long-term benefits and should be introduced cautiously. Interactions between antiretroviral drugs and other drugs, as well as kinetics, need to be taken into account. Guidelines on treatment of HIV-positive septic patients are needed.

\section{Conflict of interest}

The author's declared no potential conflicts of interest with respect to the research, authorship, and/or publication of this article.

\section{References}

1. Singer M, Deutschman CS, Seymour CW, et al. The Third International Consensus Definitions for Sepsis and Septic Shock (Sepsis-3). JAMA 2016; 315: 801-810.

2. CDC, Sepsis. CDC's National Center for Health Statistics (NCHS), Data Reports (2014); http://www.cdc.gov/sepsis/datareports/index. html. Accessed 15 June 2015.

3. Angus DC, Linde-Zwirble WT, Lidicker J, et al. Epidemiology of severe sepsis in the United States: analysis of incidence, outcome, and associated costs of care. Crit Care Med 2001; 29: 1303-1310.

4. Jawad I, Lukšić I, Rafnsson SB. Assessing available information on the burden of sepsis: global estimates of incidence, prevalence and mortality. J Glob Health 2012; 2: 010404

5. Japiassú AM, Amâncio RT, Mesquita EC, et al. Sepsis is a major determinant of outcome in critically ill HIV/AIDS patients. Crit Care 2010; 14: R152.

6. Silva JS, dos Santos S de S. Sepsis in AIDS patients: clinical, etiological and inflammatory characteristics. J Int AIDS Soc 2013; 16: 17344.

7. Casalino E, Wolff M, Ravaud P, et al. Impact of HAART advent on admission patterns and survival in HIV-infected patients admitted to an intensive care unit. AIDS 2004; 18: 1429-1433.

8. Soni N, Pozniak A. Continuing HIV therapy in the ICU. Crit Care 2001; 5: 247-248.

9. Lundgren JD, Gatell JM, Rockstroh JK, et al. European AIDS Clinical Society Guidelines version 8.0 (2015). 
10. Greenberg JA, Lennox JL, Martin GS, et al. Outcomes for critically ill patients with HIV and severe sepsis in the era of highly active antiretroviral therapy. J Crit Care 2012; 27: 51-57.

11. Petrosillo N, Pagani L, Ippolito G; Gruppo HIV e Infezioni Ospedaliere. Nosocomial infections in HIV-positive patients: an overview. Infection 2003; 31 Suppl 2: 28-34.

12. Mrus JM, Braun L, Yi M, et al. Impact of HIV/AIDS on care and outcomes of severe sepsis. Crit Care 2005; 9: R623-630.

13. Akgün KM, Tate JP, Pisani M, et al. Medical ICU admission diagnoses and outcomes in human immunodeficiency virus-infected and virus-uninfected veterans in the combination antiretroviral era. Crit Care Med 2013; 41: 1458-1467.

14. Medrano J, Álvaro-Meca A, Boyer A, et al. Mortality of patients infected with HIV in the intensive care unit (2005 through 2010): significant role of chronic hepatitis $C$ and severe sepsis. Crit Care 2014; 18: 475 .

15. Dellinger RP, Mitchell ML, Rhodes A, et al. Surviving Sepsis Campaign: International Guidelines for Management of Severe Sepsis and Septic Shock: 2012, Surviving Sepsis Campaign; http://www. sccm.org/Documents/SSC-Guidelines.pdf. Accessed 15 June 2015.

16. Hoen B, Bonnet F, Delaugerre C, et al. French 2013 guidelines for antiretroviral therapy of HIV-1 infection in adults. J Int AIDS Soc 2014; 17: 19034.

17. Lanoix JP, Andrejak C, Schimit JL, et al. Antiretroviral therapy in intensive care unit. Eur J Clin Microbiol Infect Dis 2011; 30: 1085-1093.

18. Masur H. Management of patients with HIV in the Intensive Care Unit. Proc Am Thorac Soc 2006; 3: 96-102.

19. van Lelyveld SF, Wind CM, Mudrikova T. Short- and long-term outcome of HIV-infected patients admitted to the intensive care unit. Eur J Clin Microbiol Infect Dis 2011; 30: 1085-1093.

20. Dickson SJ, Batson S, Copas AJ, et al. Survival of HIV-infected patients in the intensive care unit in the era of highly active antiretroviral therapy. Thorax 2007; 62: 964-968.

21. Chiang HH, Hung CC, Lee CM, et al. Admissions to the intensive care unit of HIV-infected patients in the era of highly active antiretroviral therapy: etiology and prognostic factors. Crit Care 2011; 15: R202.

22. Meybeck A, Lecomte L, Valette M, et al. Should highly active antiretroviral therapy be prescribed in critically ill HIV-infected patients during the ICU stay? A retrospective cohort study. AIDS Res Ther 2012; 9: 27.

23. Zolopa AR, Andersen J, Komarow L, et al. Immediate vs deferred ART in the setting of acute AIDS-related opportunistic infection: Final results of a randomized strategy trial, ACTG A5164. In 15th Conference on Retroviruses and Opportunistic Infections, Boston MA, USA, 2008.

24. Orsini J, Ahmad N, Butala A, et al. Etiology and Outcome of Patients with HIV Infection and Respiratory Failure Admitted to the Intensive Care Unit. Interdiscip Perspect Infect Dis 2013; 2013 732421. 